This document is confidential and is proprietary to the American Chemical Society and its authors. Do not copy or disclose without written permission. If you have received this item in error, notify the sender and delete all copies.

\title{
On-surface Synthesis of Rylene-type Graphene Nanoribbons
}

\begin{tabular}{|c|c|}
\hline Journal: & Journal of the American Chemical Society \\
\hline Manuscript ID: & ja-2014-11995r.R2 \\
\hline Manuscript Type: & Communication \\
\hline Date Submitted by the Author: & 12-Mar-2015 \\
\hline Complete List of Authors: & $\begin{array}{l}\text { Zhang, Haiming; Soochow University, Institute of Functional Nano \& Soft } \\
\text { Materials (FUNSOM) } \\
\text { Lin, Haiping; Soochow University, (FUNSOM) Institute of Functional } \\
\text { Nano\&Soft Materials } \\
\text { Sun, Kewei; FUNSOM, Soochow University } \\
\text { Chen, Long; Tianjin University, Department of Chemistry } \\
\text { Zagranyarski, Yulian; Max-Planck-Institute for Polymer Research, } \\
\text { Aghdassi, Nabi; Soochow University, FUNSOM } \\
\text { Duhm, Steffen; Soochow University, FUNSOM } \\
\text { Li, Qing; Institute of Functional Nano \& Soft Materials, } \\
\text { Zhong, Dingyong; School of Physics and Engineering \& State Key } \\
\text { Laboratory of Optoelectronic Materials and Technologies, Sun Yat-sen } \\
\text { University } \\
\text { Li, Youyong; Soochow University, Institute of Functional Nano \& Soft } \\
\text { Materials (FUNSOM) } \\
\text { Müllen, Klaus; Max-Planck-Institute for Polymer Research, } \\
\text { Fuchs, Harald; Westfalische Wilhelms-Universitaet, Physikalisches Institut } \\
\text { Chi, Lifeng; University Muenster, ; FUNSOM, Soochow University }\end{array}$ \\
\hline
\end{tabular}

\section{SCHOLARONE ${ }^{\text {M }}$}

Manuscripts 
Lateral quantum confinement opens the band gap of graphene nanoribbons (GNRs) making it an attractive semiconductor material. Since the edge geometry and the width of GNRs strongly affect their energy band structures, ${ }^{1-5}$ preparing GNRs with atomic precision is crucial for their future applications. While top-down fabrication pathways cannot fully match these requirements, ${ }^{6-8}$ recent progress in bottom-up synthesis of armchair graphene nanoribbons (AGNRs) on single crystal metal surfaces holds great promise to achieve the desired structural perfection. ${ }^{9-16}$ For instance, 7-AGNRs ${ }^{9}$ and ${ }^{13}$-AGNRs ${ }^{14}$ (the integer refers to the number of carbon atoms across the width of a GNR) were synthesized on $\mathrm{Au}(111)$ surfaces via a two-step reaction, i.e. thermally initiated dehalogenation and following dehydrogenation at a higher substrate temperature (ca. 67oK). The synthesized atomically precise AGNRs further facilitate the experimental measurement on its electronic structures by means of photoemission spectroscopy (PES) ${ }^{11}$ as well as scanning tunneling spectroscopy (STS) ${ }^{13-15}$ As theoretically predicted, the electronic structure of N-AGNRs can be divided into three subfamilies with $\mathrm{N}=3 \mathrm{p}, 3 \mathrm{p}+1$ and $3 \mathrm{p}+2 \quad(\mathrm{p}=1,2,3 \cdots)$, because of the various boundary conditions. ${ }^{2}$ Specifically, n-

\begin{abstract}
The narrowest armchair graphene nanoribbon (AGNR) with five carbons across the width of the GNR (5AGNR) was synthesized on $\mathrm{Au}(111)$ surfaces via sequential dehalogenation processes in a mild condition by using 1,4,5,8-tetrabromonaphthalene (TBN) as the molecular
precursor. Gold-organic hybrids were observed by using high resolution scanning tunneling microscopy (STM) and
considered as intermediate states upon AGNR formation. Scanning tunneling spectroscopy (STS) reveals an unexpectedly large band gap of $\Delta=2.8 \pm 0.1 \mathrm{eV}$ on $\mathrm{Au}(111)$
surface which can be interpreted by the hybridization of the surface states and the molecular states of the 5-AGNR.
\end{abstract}

AGNRs with $n=3 p+2$ are predicted to be metallic by tightbinding approximations, ${ }^{3}$ or semiconducting by density functional theory (DFT) calculations. ${ }^{2}$ However, the band gaps of $n$-AGNRs with $n=3 p+2$ have been rarely explored experimentally, due to the lack of materials with well-defined structures.

Herein, we report the synthesis of 5-AGNRs, the narrowest $\mathrm{n}$-AGNRs in subfamily of $\mathrm{n}=3 \mathrm{p}+2$, on $\mathrm{Au}(111)$ surfaces via dehalogenation of 1,4,5,8-tetrabromonaphthalene (TBN) under ultra-high vacuum (UHV). Scanning tunneling microscopy (STM) reveals the structural evolution of the ribbons upon substrate temperature, revealing for the first time the intermediate Au-naphthalene hybrids toward the formation of 5-AGNRs. In addition, since no dehydrogenation step is involved, the preparation temperature is thus significantly reduced. The resulting ultra-narrow ribbon with a rylene (i.e. fused oligo(peri-naphthalene)s) structure ${ }^{17}$ is shown by STS to have an unexpected larger band gap of $2.8 \pm 0.1 \mathrm{eV}$ in comparison with that of 7 -AGNR $(2.5 \pm 0.1 \mathrm{eV}$ by STS $){ }^{11,12,14}$ which is significantly larger than the theoretically predicted one without metal support.

A typical STM image with intact molecules evaporated on a $\mathrm{Au}(111)$ surface at a temperature below $273 \mathrm{~K}$ is shown in Figure 1a. Each bump represents a single TBN molecule. According to the measured periodic parameters of $a=0.90 \pm$ $0.02 \mathrm{~nm}$ and $\mathrm{b}=1.60 \pm 0.04 \mathrm{~nm}$, a structural model has been established (inset, Figure 1a). Deposition of TBN molecules on hot $\mathrm{Au}(111)$ surfaces promotes the dissociation of $\mathrm{C}-\mathrm{Br}$ bonds and the subsequent coupling of TBN radicals. ${ }^{18}$ Figure $\mathrm{lb}$ depicts a STM image of the sample prepared by depositing TBN on the $\mathrm{Au}(111)$ surface held at $370 \mathrm{~K}$. Next to some isolated ribbons, the $\mathrm{Au}(111)$ surfaces are mainly covered by islands of monolayers with structural details shown in Figure 1c. A rectangular unit cell with parameters of $a=1.00 \pm 0.02$ $\mathrm{nm}$ and $\mathrm{b}=1.19 \pm 0.03 \mathrm{~nm}$ differs from that of TBN SAMs. Since the size of the molecule is remarkably larger than that of a TBN molecule and the substrate temperature $(370 \mathrm{~K})$ is high enough to dissociate $\mathrm{C}-\mathrm{Br}$ bonds, ${ }^{19}$ an organogold 
compound is proposed and overlaid on the image for clarity. The chemical structure of the organogold compound has been confirmed by complementary X-ray photoemission spectroscopy (XPS) measurements and DFT simulations of STM images (see Figure S1). Dark spots appear periodically along the [11-2] direction in the SAMs of organogold compound (Figure 1c, labeled by a black star), representing the empty space between two organogold compounds.
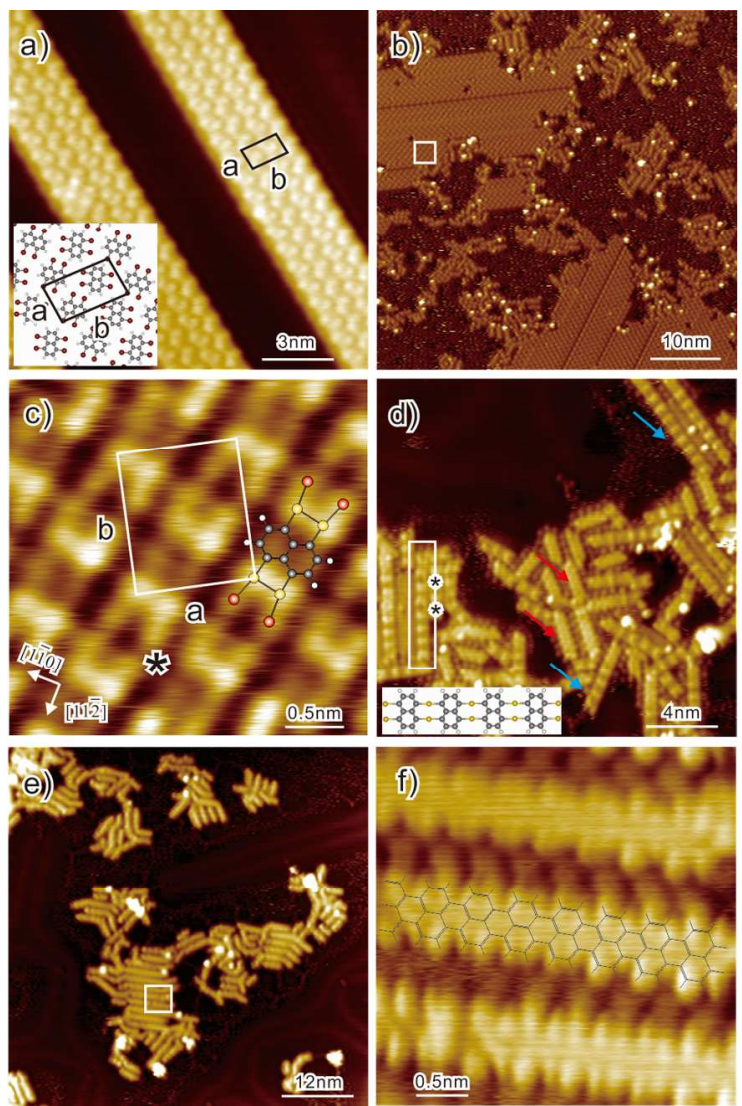

Figure 1. Synthesis of 5-AGNRs on Au(111) surfaces. a) STM image of the TBN SAMs. Inset: The corresponding structural model for TBN SAMs. b) STM image of the sample prepared by depositing TBN at the $\mathrm{Au}(111)$ surface held at $37 \mathrm{OK}$. c) Atomically high resolution STM image of organogold intermediate. A chemical structure of Au-naphthalene compound is overlaid for a better illustration (yellow: $\mathrm{Au}$, red: $\mathrm{Br}$, gray: $\mathrm{C}$, white: $\mathrm{H})$. d) STM image of the $\mathrm{Au}(111)$ surface covered by abundant dinaphthyl-aurate chains. The sample is obtained by depositing TBN on the Au(111) surfaces held at $400 \mathrm{~K}$. A white rectangle highlights a ribbon, in which differences between linearly packed organogold compounds and a dinaphthyl-aurate chain are labeled by two black stars. Inset: the chemical structure of a dinaphthyl-aurate chain. e) STM image of 5-AGNRs fabricated by further annealing the sample at 470K. f) High resolution STM image of 5-AGNR. The chemical structure of a 5 -AGNR is overlaid on the image.

When TBN molecules were deposited on the Au(111) surface held at a higher temperature (e.g. 40oK), the amount of the initial organogold species was reduced remarkably by STM investigations at $77 \mathrm{~K}$. Although a few organogold molecules, featured with similar dark spots as shown in Figure 1c, are still observable (see the upper part highlighted by a white rectangle in panel $d$ ), the major parts of the

surface are covered by ribbons. The ribbons can be divided into two co-existing structures at this substrate temperature (Figure $1 \mathrm{~d}$ ): one exhibits a nodal feature (blue arrows), and the other is more flat (red arrows). The profile of the "nodal ribbon" reveals a periodic distance of $0.72 \pm 0.02 \mathrm{~nm}$, which fits well to that of covalently connected bis(1,8-naphthylene) diaurate (inset, Figure 1d). Since the naphthalene core remains almost unchanged, the $\mathrm{Au}-\mathrm{C}$ distance can be derived to be $2.2 \pm 0.2 \AA$, which is consistent with the Au-C bonding length reported in the literature. ${ }^{20}$ We have already demonstrated that radicals formed by the dissociation of $\mathrm{C}$ $\mathrm{Cl}$ bonds can covalently connect to gold atoms forming stable gold-organic hybrids on $\mathrm{Au}(111)$ surfaces. $^{21}$ It is thus reasonable that the "nodal ribbon" is composed of covalently connected Au-naphthalene complexes (inset of Figure 1d). Further annealing at $470 \mathrm{~K}$ transforms the "nodal ribbons" into the "flat ribbons" (Figure 1e). A high-resolution STM image (Figure if) discloses that the periodic distance along the ribbon $(0.42 \pm 0.01 \mathrm{~nm})$ agrees well with the periodic distance of 5-AGNR.

b)

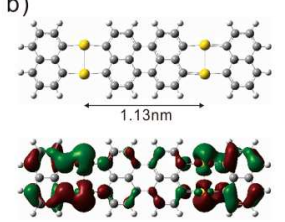

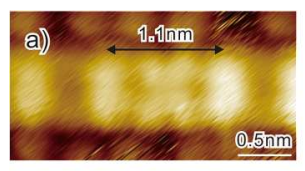

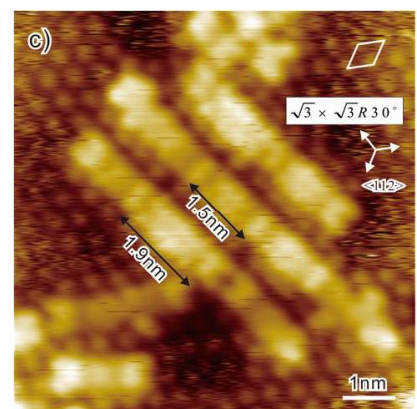

Figure 2. The existence of Au-naphthylene intermediate in synthesis of 5 -AGNRs. a) High resolution STM image of a dinaphthyl-aurate chain containing a dimer of covalently connected naphthylene (Au-di-nap). b) Optimized geometric structure (top) and molecular orbitals (bottom) of a molecule containing a Au-di-nap structure. The distance marked in the image $(1.13 \mathrm{~nm})$ is consistent with the measured distance from STM image. c) STM image of Aunaphthylene intermediate.

To support the existence of dinaphthyl-aurate intermediates, high resolution STM images were recorded with the sample held at 40oK. Figure $2 \mathrm{a}$ presents the STM image of a dinaphthyl-aurate chain containing a dimer of naphthylene (hereafter, Au-di-nap) with a length $1.12 \pm 0.02$ $\mathrm{nm}$ (from $\mathrm{Au}$ to $\mathrm{Au}$ ) which is consistent with that of an optimized geometric structure (top, Figure $2 \mathrm{~b}$ ). The shape of the dimer in the STM image is featured by star-shaped four lobs which resemble the calculated highest occupied molecular orbital (HOMO) of the Au-di-nap (bottom, Figure 2b). Other Au-naphthylene intermediates with various length of rylene-type ribbon have also been observed from STM investigations, for instance, intermediates with lengths of $1.5 \mathrm{~nm}$ and $1.9 \mathrm{~nm}$, corresponding to three and four covalently connected naphthalene moieties, respectively (see Figure $2 \mathrm{c}$ and supporting materials for optimized geometric structures by DFT simulations, Figure S2). Hexagonally packed bright bumps with $\sqrt{3} \times \sqrt{3} R_{30}$ unit cell (as marked in Figure $2 \mathrm{c}$ ) are attributed to chemisorbed $\mathrm{Br}$ radicals, in accordance with the literature. ${ }^{22}$ 
Covalently connected metal-organic hybrids as intermediate states in the surface supported aryl-aryl coupling have been theoretically predicted and experimentally verified by STM investigations on $\mathrm{Cu}$ (111) and $\mathrm{Ag}$ (111) surfaces. $^{23-25}$ However, stable gold-organic intermediate states in "Ullmann type" aryl-aryl couplings on gold surfaces have rarely been reported. ${ }^{9,26}$ Our previous work reveals the existence of stable gold-organic hybrids in the surfaceassisted reaction of perylene derivatives with the chloro substituents in the bay region, but the reaction does not proceed beyond this stage. ${ }^{21}$ By further increasing the substrate temperature, the gold-organic hybrids decomposed. In contrast, the Au-naphthalene hybrids obtained here are obviously the intermediate states toward the formation of 5AGNRs, indicating a possible pathway for Ullmann coupling reactions on $\mathrm{Au}(111)$ surfaces.

Since $\mathrm{Br}$ radicals generated by $\mathrm{C}-\mathrm{Br}$ bond dissociation may chemisorb on $\mathrm{Au}(111)$ surfaces surrounding 5-AGNRs (see Figure 2c), the sample was annealed at a higher temperature (ca. $570 \mathrm{~K}$ ). Shown in Figure $3 \mathrm{a}$ is the high resolution STM image of a 5 -AGNR without surrounding $\mathrm{Br}$ radicals (see also Figure $S_{3}$ ). STS measurements of 5 -AGNRs are performed on more than ten ribbons with various lengths (from $4 \mathrm{~nm}$ up to 2onm). The typical $\mathrm{d} I / \mathrm{d} V$ curve on a 5 -AGNR is depicted in Figure $3 \mathrm{~b}$ (blue line), whereas a curve on a bare $\mathrm{Au}(111)$ surface is provided for comparison (red line). The surface state of $\mathrm{Au}(111)$ with an onset at $-0.5 \mathrm{~V}$ is obtained on the bare $\mathrm{Au}(111)$ surface which contributes to the resonance in the $\mathrm{d} I / \mathrm{d} V$ spectrum of 5 -AGNR with a broad peak roughly between $-0.4 \mathrm{~V}$ and $0.6 \mathrm{~V}$. A similar feature in the $\mathrm{d} I / \mathrm{d} V$ spectrum is also found for 7 -AGNR (Figure $\mathrm{S}_{4}$ ). ${ }^{13}$ Apart from this broad peak originating from the surface states, the evident difference in $\mathrm{d} I / \mathrm{d} V$ spectra between the 5-AGNR and the bare $\mathrm{Au}(111)$ reflects the electronic properties of 5-AGNR. Two clear features appear in the 5 -AGNR $\mathrm{d} I / \mathrm{d} V$ curve with 2.1 $\mathrm{V}$ and $-0.65 \mathrm{~V}$ for the unoccupied state and the occupied state, respectively. Statistical analysis of overall 5-AGNR $\mathrm{d} I / \mathrm{d} V$ spectra (see Figure $\mathrm{S}_{5}$ and $\mathrm{S} 6$ for more evidences) provides an average value of $2.07 \pm 0.05 \mathrm{eV}$ and $-0.69 \pm 0.06$ $\mathrm{eV}$ for the empty state and the filled state, respectively, yielding a band gap of $\Delta=2.8 \pm 0.1 \mathrm{eV}$.

Further measurements on the spatial distribution of the electronic structure of 5 -AGNRs are conducted by grid $\mathrm{d} I / \mathrm{d} V$ measurements across the ribbon as well as constant-bias $\mathrm{d} I / \mathrm{d} V$ mapping at various sample bias. A $2 \mathrm{D}$ STS map (Figure 3c) composed of $45 \mathrm{~d} I / \mathrm{d} V$ spectra recorded along the white line marked in the inset of Figure $3 \mathrm{~b}$ indicates the edgedependent distribution of the empty state (ca. 2.1 V) and the filled state (ca. - $0.7 \mathrm{~V}$ ). Besides the two states mentioned above, no other significant features were found on the ribbon. The overall distribution of each state on the 5-AGNR is depicted in $\mathrm{d} I / \mathrm{d} V$ mapping at a sample bias corresponding to the valence (Figure $3 \mathrm{~d}$ ) and conduction band edges (Figure 3f), respectively. The enhanced intensity along the two edges of the ribbon indicates the spatial distribution of these energies with a higher local density of state (LDOS) as compared to the remaining part of the ribbon. In contrast, the $\mathrm{d} I / \mathrm{d} V$ map recorded at a sample bias of $0.5 \mathrm{~V}$ (Figure $3 \mathrm{e}$ ) appears dark at the edge of the ribbon, presenting an example of spatial distribution of energies within the band gap.
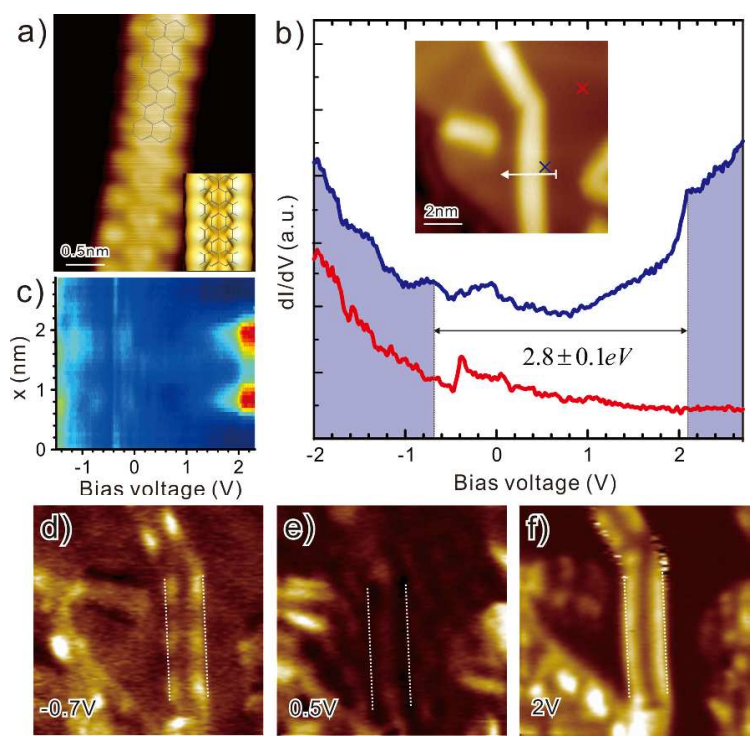

Figure 3. STS measurement ( $\mathrm{d} I / \mathrm{d} V$ spectra) of the band gap of 5 -AGNR. a) High resolution STM image of a 5-AGNR. The chemical structure of 5 -AGNR is overlaid on the image for better illustration. Inset: DFT simulated STM image of 5AGNR on the $\mathrm{Au}(111)$ surface. b) $\mathrm{d} I / \mathrm{d} V$ spectra obtained on a bare $\mathrm{Au}(111)$ surface (red line) and on the edge of 5-AGNR (blue line). The spectrum of 5-AGNR is offset vertically for clarity. c) $2 \mathrm{D}$ STS map composed of $45 \mathrm{~d} I / \mathrm{d} V$ spectra recorded across the 5-AGNR along the white line marked in panel b. d)-f) $\mathrm{d} I / \mathrm{d} V$ maps of 5-AGNR obtained at bias voltage of $-0.7 \mathrm{~V}(\mathrm{~d}), 0.5 \mathrm{~V}(\mathrm{e})$ and $2 \mathrm{~V}(\mathrm{f})$, respectively.

The 5-AGNRs belong to the sub-family of n-AGNRs $(\mathrm{n}=3 \mathrm{p}+2$, with $\mathrm{p}=1)$ which differ from previously synthesized 7-AGNRs and 13-AGNRs that are members of the sub-family with $n=3 p+1$. The band gaps of $n-A G N R s$ with $n=3 p+2$ are theoretically predicted to be lower than those of n-AGNRs of $\mathrm{n}=3 \mathrm{p}$ and $3 \mathrm{p}+1 .^{1-3}$ Calculations of isolated 5-AGNRs with many-body perturbation theory ( $G W$ approximation, $\mathrm{G}=\mathrm{Green}$ 's function; $\mathrm{W}=$ screened Coulomb interaction) provide a band gap of $1.32 \mathrm{eV}^{27}$ which is significantly lower than that $(2.8 \pm 0.1 \mathrm{eV})$ obtained from STS measurements conducted here on the $\mathrm{Au}$ surface. Since band gap predictions still remain a challenge in DFT simulations, we adopt the value obtained by $G W$ approximation as reference which is currently the most accurate method in predicting the band gap of a semiconductor. However, as the computational costs required for $G W$ calculations are much higher than those of typical DFT calculations, substrate effects are rarely involved in the calculations. The band gaps measured by STS (2.5 eV for $7-\mathrm{AGNR}^{13}$ and $1.4 \mathrm{eV}$ for $13-$ $\mathrm{AGNR}^{14}$ ) are consistently narrower than those predicted by $G W$ approximations (3.8 eV for 7-AGNR and $2.4 \mathrm{eV}$ for 13AGNR). However, in the case of 5-AGNR, the band gap from STS is unexpectedly larger in comparison with theoretical predictions. This striking finding might originate from the interactions between 5 -AGNR and the $\mathrm{Au}(111)$ substrate or a local electric field introduced by the STM tip, ${ }^{28}$ as the electric field is expected to have a much stronger influence on narrower ribbons. A similar broadening of the band gap has also been observed in STS spectra of a polyfluorene chain on $\mathrm{Au}(111) .{ }^{29}$ To understand the band broadening of the 5-AGNR supported on a $\mathrm{Au}(111)$ substrate, we have performed first- 
principles STS calculations with the Tersoff-Hamann's approach (without STM tip) and the Bardeen's theory (the STM tip was simulated with an ideal tungsten pyramid), respectively. Our theoretical results show that the adsorption of 5-AGNRs on the Au(111) surfaces can lead to the broadening of the band gap. Such a broadening effect can be interpreted by the hybridization of the surface states and the molecular states of the 5-AGNR (see Figure $S_{7}$ and S8), which differs from the weakly hybridized systems, such as $7^{-}$ AGNRs/Au(111). ${ }^{13}$

In summary, we reported the synthesis of narrowest GNRs on $\mathrm{Au}(111)$ surface via sequential dehalogenation processes by using TBN molecules as precursors. Gold-organic hybrids have been observed by atomically high resolution STM images and they are considered as intermediate states in synthesis of 5-AGNRs on $\mathrm{Au}(111)$ surfaces. The stable structure of bis(1,8-naphthylene)diaurate ensures the correct carbon-carbon connection to form graphene nanoribbons. STS measurements on 5-AGNRs supported on Au(111) surface reveal a band gap of $\Delta=\mathbf{2 . 8} \pm 0.1 \mathrm{eV}$, which is significantly larger than the value $(1.32 \mathrm{eV})$ predicted by $\mathrm{GW}$ approximation of isolated 5-AGNR. The band gap measurements of 5-AGNRs will undoubtedly promote further research on energy band structure determinations of other nAGNRs in the same family with $\mathrm{n}=3 \mathrm{p}+2$.

\section{Supporting Information}

Experimental procedures, simulation details, XPS results, additional STM $\mathrm{d} I / \mathrm{d} V$ spectrum analysis of 5 -AGNR, DFT calculations on the adsorption induced broadening of band gap, synthesis of TBN, and Figures S1-S1o. This material is available free of charge via the Internet at http://pubs.acs.org.

\section{AUTHOR INFORMATION}

Corresponding Author

Email: chilf@suda.edu.cn, muellen@mpip-mainz.mpg.de fuchsh@uni-muenster.de.

\section{ACKNOWLEDGMENT}

This work was supported by the National Natural Science Foundation of China (NSFC, Grant No. 91227201) and German Science Foundation (DFG, TRR61, SFB858 and SPP1459. We also thank the Collaborative Innovation Center of Suzhou Nano Science \& Technology, and the Priority Academic Program Development of Jiangsu Higher Education Institutions, the European Research Council grant on NANOGRAPH, Graphene Flagship (No. CNECT-ICT604391), the Major State Basic Research Development Program of China (973 Program, Nos. 2013CB93350o and 2014CB9326oo), NSFC (Grant No. 21403149, 91233115, 21273158) and European Union Projects UPGRADE, GENIUS, and MoQuaS.

\section{REFERENCES}

(1)Nakada, K.; Fujita, M.; Dresselhaus, G.; Dresselhaus, M. S. Phys. Rev. B 1996, 54, 17954-17961.
(2)Son, Y. W.; Cohen, M. L.; Louie, S. G. Phys. Rev. Lett. 20o6, 97, 216803.

(3)Wakabayashi, K.; Fujita, M.; Ajiki, H.; Sigrist, M. Phys. Rev. B 1999 59, 8271-8282.

(4)Han, M. Y.; Ozyilmaz, B.; Zhang, Y. B.; Kim, P. Phys. Rev. Lett. 2007, 98, 206805.

(5)Kobayashi, Y.; Fukui, K.; Enoki, T.; Kusakabe, K.; Kaburagi, Y. Phys. Rev. B 2005, 71, 193406.

(6)Kosynkin, D. V.; Higginbotham, A. L.; Sinitskii, A.; Lomeda, J. R.; Dimiev, A.; Price, B. K.; Tour, J. M. Nature 2009, 458, 872-U5.

(7)Li, X.; Wang, X.; Zhang, L.; Lee, S.; Dai, H. Science 20o8, 319, 12291232.

(8)Wang, X. R.; Ouyang, Y. J.; Jiao, L. Y.; Wang, H. L.; Xie, L. M.; Wu, J.; Guo, J.; Dai, H. J. Nature Nanotechnol. 2o11, 6, 563-567.

(9)Cai, J. M.; Ruffieux, P.; Jaafar, R.; Bieri, M.; Braun, T.; Blankenburg, S.; Muoth, M.; Seitsonen, A. P.; Saleh, M.; Feng, X. L.; Mullen, K.; Fasel, R. Nature 2010, 466, 470-473.

(10)Koch, M.; Ample, F.; Joachim, C.; Grill, L. Nature Nanotechnol. 2012, 7, 713-717.

(11)Linden, S.; Zhong, D.; Timmer, A.; Aghdassi, N.; Franke, J. H.; Zhang, H.; Feng, X.; Müllen, K.; Fuchs, H.; Chi, L.; Zacharias, H. Phys. Rev. Lett. 2012, 108, 216801.

(12)Talirz, L.; Sode, H.; Cai, J. M.; Ruffieux, P.; Blankenburg, S.; Jafaar, R.; Berger, R.; Feng, X. L.; Müllen, K.; Passerone, D.; Fasel, R.; Pignedoli, C. A. J. Am. Chem. Soc. 2013, 135, 2060-2063.

(13)Ruffieux, P.; Cai, J. M.; Plumb, N. C.; Patthey, L.; Prezzi, D.; Ferretti, A.; Molinari, E.; Feng, X. L.; Müllen, K.; Pignedoli, C. A.; Fasel, R. Acs Nano 2012, 6, 6930-6935.

(14)Chen, Y. C.; de Oteyza, D. G.; Pedramrazi, Z.; Chen, C.; Fischer, F. R.; Crommie, M. F. Acs Nano 2013, 7, 6123-6128.

(15)Huang, H.; Wei, D.; Sun, J.; Wong, S. L.; Feng, Y. P.; Neto, A. H. C.; Wee, A. T. S. Sci. Rep. 2012, 2, 983.

(16)Sakaguchi, H.; Kawagoe, Y.; Hirano, Y.; Iruka, T.; Yano, M.; Nakae, T. Adv.Mat. 2014, 26,4134.

(17)Bohnen, A.; Koch, K.-H.; Lüttke, W.; Müllen, K. Angew. Chem. Int. Ed. 1990, 29, 525-527.

(18)Franc, G.; Gourdon, A. Phys. Chem. Chem. Phys. 2o11, 13, 1428314292.

(19) (a) Batra, A; Cvetko, D.; Kladnik, G.; Adak, O.; Cardoso, C.; Ferretti, A.; Prezzi, D.; Molinari, E.; Morgante, A.; Venkataraman, L. Chem. Sci., 2014, 5, 4419. (b) Simonov, K. A.; Vinogradov, N. A.; Vinogradov, A. S.; Generalov, A. V.; Zagrebina, E. M.; Mårtensson, N.; Cafolla, A. A.; Carpy, T.; Cunniffe, J. P.; Preobrajenski, A. B. J. Phys. Chem. C 2014, 118, 12532-12540.

(20)Fernandez, E. J.; Jones, P. G.; Laguna, A.; Lopez-de-Luzuriaga, J. M.; Monge, M.; Perez, J.; Olmos, M. E. Inorg. Chem. 2002, 41, 1056. (21)Zhang, H.; Franke, J.-H.; Zhong, D.; Li, Y.; Timmer, A.; Arado, O. D.; Mönig, H.; Wang, H.; Chi, L.; Wang, Z.; Müllen, K.; Fuchs, H. Small 2014, 10, 1361.

(22)Tao, N. J.; Lindsay, S. M., J. Phys. Chem. 1992, 96, 5213-5217.

(23)Bieri, M.; Nguyen, M.-T.; Gröning, O.; Cai, J.; Treier, M.; AÏtMansour, K.; Ruffieux, P.; Pignedoli, C. A.; Passerone, D.; Kastler, M.; Müllen, K.; Fasel, R. J. Am. Chem. Soc. 2o1o, 132, 16669-16676. (24)Nguyen, M. T.; Pignedoli, C. A.; Passerone, D. Phys. Chem. Chem. Phys. 2011, 13, 154-16o.

(25)Wang, W. H.; Shi, X. Q.; Wang, S. Y.; Van Hove, M. A.; Lin, N. J. Am. Chem. Soc. 2011, 133, 13264-13267.

(26)Grill, L.; Dyer, M.; Lafferentz, L.; Persson, M.; Peters, M. V.; Hecht, S. Nature Nanotechnol. 2007, 2, 687-691.

(27)Yang, L.; Park, C. H.; Son, Y. W.; Cohen, M. L.; Louie, S. G. Phys. Rev. Lett. 2007, 99, 186801.

(28) Binnig, G.; Frank, K. H.; Fuchs, H.; Garcia, N.; Reihl, B.; Rohrer, H.; Salvan, F.; Williams, A. R. Phys. Rev. Lett. 1985, 55, 991.

(29) Bombis, C.; Ample, F.; Lafferentz, L.; Yu, H.; Hecht, S.; Joachim, C.; Grill, L. Angew. Chem. Int. Ed. 2009, 48, 9966-9970. 


\section{Page 5 of 5}

1

2

3

4

5

6

7

8

9

10

11

12

13

14

15

16

17

18

19

20

21

22

23

24

25

26

27

28

29

30

31

32

33

34

35

36

37

38

39

40

41

42

43

44

45

46

47

48

49

50

51

52

53

54

55

56

57

58

59

60

TOC
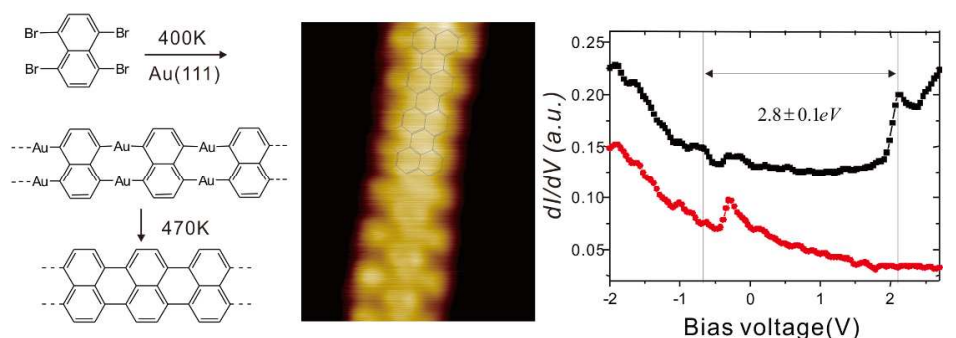